Berkeley the hottest debates were those between C. D. Bailyn (Yale University) and S. Kulkarni (California Institute of Technology) on this issue.

The main lesson to be learned from these developments is that the system of globular clusters is like a galactic ecosystem. None of the important issues can be considered in isolation, because they are so closely interlinked: the galactic gravitational field influences the dynamical evolution, the evolution influences the stellar density, and this in turn affects the ways in which individual stars and binary stars can evolve. Several pieces of this web of interactions have become clearer in recent years, and one of the most exciting challenges in the coming decade will be the task of delineating the entire network.

Douglas C. Heggie is in the Department of Mathematics and Statistics, University of Edinburgh, Edinburgh EH9 3JZ, UK.

\title{
Progress by poisoning
}

\section{Dan Cutler}

ExoTIC poisons have often been intrumental in revealing cellular and molecular phenomena. A new and particularly striking example is to be found in the paper by Schiavo et al. on page 832 of this issue ${ }^{1}$. The authors describe experiments which show that tetanus toxin $(\mathrm{TeTx})$ and botulinum-B toxin (BoNT) exert their deadly effects through the cleavage of one particular synaptic vesicle membrane protein, synaptobrevin- 2 .

These neurotoxins are structurally similar and are each synthesized as a precursor with low toxicity, which is processed to the active form of two disulphide-linked subunits. One subunit (the heavy chain) mediates binding to the cell surface, conferring target specificity, and facilitates entry of the other subunit (the light chain) into the cytosol where it causes the toxic effect. The different clinical effects of the two toxins reflect their different target cells: TeTx causes spastic paralysis by blocking presynaptic neurotransmitter release in the central nervous system, whereas BoNT causes flaccid paralysis by blocking acetylcholine release at the neuromuscular junction.

Recently, Schiavo et al. and others looked into the possibility - initially suggested by protein sequence data that both toxins are zinc-dependent endopeptidases. The conserved zincbinding motif characteristic of this activity $^{2}$ seems to be functionally important; TeTx (refs 3, 4) and BoNT (ref. 5) light chains both contain zinc. Chelation of the ion both blocks the toxic effect of TeTx on Aplysia neurons injected with toxin, and inhibits the proteolytic activity of the light chain in vitro ${ }^{4}$.

What is the target of this proteolytic activity? Schiavo et al. have now explored the possibility that it lies in the synaptic vesicle membrane. They incubated highly purified synaptic vesicles with the toxins and show that both cleave a membrane protein with the electrophoretic mobility of synaptobrevin-2 (ref. 6). The cleavage produces two fragments with a time course matching that of the inhibition of neurotransmitter release in Aplysia neurons injected with toxin. Protein sequencing confirms that the protein affected is synaptobrevin-2, and that both toxins cleave at the same single peptide bond. A peptide corresponding to the cleavage site can also inhibit toxin function both in vivo and in vitro. So it seems that TeTx and BoNT block transmitter release by cleavage of synaptobrevin-2, and that this is the only target for these toxins.

Apart from the satisfaction of identifying the molecular target of two neurotoxins whose effects have been known for such a long time (the symptoms of tetanus poisoning were described by Hippocrates), these are the first toxins to be shown to exert their actions through effects on a synaptic vesicle membrane protein. For those in the business this is an exciting development - work on this membrane is already intense, and further clues to the function of its proteins will add impetus to the field.

The identification and characterization of the protein components of the synaptic vesicle membrane is proceeding at such a pace that it may become the first organelle for which all the integral membrane protein components are described. They may be divided into two main categories ${ }^{7}$ : components that carry out the uptake and storage of neurotransmitter; and components that include those responsible for the membrane traffic of synaptic vesicles, including targeting, docking and fusion at the presynaptic plasma membrane, as well as the subsequent retrieval and reconstitution of the vesicle. These events also occur during vesicular traffic between other membranes, but the proteins under consideration are confined to neurons and neuroendocrine cells. They must therefore carry out aspects of these tasks particularly associated with neural and neuroendocrine secretion, including the regulation of secretion in response to external secretagogue action, and the tight docking of synaptic vesicles at the plasma membrane (thought to contribute to the extremely rapid exocytic response to stimulation).

It is proving difficult to assign specific functions to members of this group of proteins, although there has been some progress. Of the three main members, the synaptotagmins (p65) have been implicated in synaptic vesicle docking and fusion $^{7}$ (although a recent paper describes a neuroendocrine cell line without p65 that still has a fully functional regulated secretory pathway ${ }^{8}$ ), and synaptophysin (p38) is a candidate for the exocytic pore complex ${ }^{7}$. But there have been few clues to the function of the synaptobrevins, and the unequivocal demonstration that the proteolytic cleavage of synaptobrevin-2 abolishes synaptic vesicle function is a major step forward.

The findings of Schiavo et al. raise many questions. The structure of synaptobrevin-2, with the bulk of the protein on the cytoplasmic side of the membrane, suggests that it interacts either with the plasma membrane or with some cytoplasmic molecule. But if synaptotagmin is involved in docking, what might synaptobrevin- 2 be needed for? If it is involved in targeting, then where do the synapsins, which mediate interactions between the vesicle and the cytoskeleton ${ }^{9}$, fit in? Whether this apparent confusion of overlapping functions reflects the extensive (and possibly cooperative) interactions that occur ${ }^{10}$ between synaptic vesicle membrane proteins, or whether we do not yet appreciate the full range of tasks carried out by this membrane, is not clear. But one thing is certain: identification of a few more toxins whose target lies within the synaptic vesicle would be very useful. In the meantime, sales of tetanus toxin and botulinum-B toxin will surely rise.

Dan Cutler is in the MRC Laboratory of Molecular Cell Biology, University College London, Gower Street, London WC1E 6BT, UK.

1. Schiavo, G. et al. Nature 359, 832-835 (1992)

2. Jongeneel, C. V., Bouvier, J. \& Bairoch, A. FEBS Lett. 242, 211-214 (1989)

3. Wright, J. F., Pernollet, M., Reboul, A., Aude, C. \& Colomb, M. G. J. biol. Chem. 267, 9053-9058 (1992).

4. Schiavo, G. et al. EMBO J. 11, 3577-3583 (1992).

5. Schiavo, G. Rossetto, O. Santucci, A. Dasgupta, B. R. Montecucco, C. J. biol. Chem. (in the press).

em. (in the press)

. Elferink, L. A., Trimble, W. S. \& Scheller, R. H. J. biol. Chem. 264, 11061-11064 (1989)

7. Sudhof, T. C. \& Jahn, R. Neuron 2, 665-677 (1991).

8. Shoji-Kasai, Y. et al. Science 256, 1820-1823 (1992).

9. Valtorta, F. et al. J. biol. Chem. 264, 7195-7198 (1992).

10. Bennett, M. K., Calakos, N., Kreiner, T. \& Scheiller, R. H. J. Cell Biol. 116, 761-775 (1992). 